\title{
Impact of an interstitial lung disease service in the diagnosis and management of interstitial lung disease in Singapore
}

Gin Tsen $\underline{\text { Chai }}{ }^{1}$, MBBS, MRCP, Teck Choon $\underline{T^{2}{ }^{2}}$, MBBS, MRCP, Yeong Shyan $\underline{\text { Lee }}{ }^{3}$, MBBCh, FRCR, Gregory JL $\underline{K a w}^{3}$, MBBS, FRCR, Khoon Leong $\underline{\mathrm{Chuah}^{4}}$, MBBS, FRCPA, Yi Jing $\underline{\mathrm{Lim}}^{5}$, BSc, PharmD, John Arputhan Abisheganaden ${ }^{1}$, MBBS, FRCP, Bernard YH Thong ${ }^{6}$, MBBS, FRCP

INTRODUCTION The current gold standard for diagnosing interstitial lung disease (ILD) involves an ILD clinic evaluation, followed by discussion in a multidisciplinary meeting (MDM). However, there is a paucity of data on the impact of ILD MDMs on the diagnosis and management of ILDs in Southeast Asia. We studied the clinical impact of the ILD service on the diagnosis and management of ILDs at a university-affiliated tertiary hospital in Singapore.

METHODS A single-centre retrospective review was done on 97 consecutive patients referred for evaluation to the ILD service from March 2016 to August 2017.

RESULTS Mean age of the patients was $67 \pm 11$ years. Gender distribution was almost equal (52\% male), with a majority of never-smokers (63\%). Mean forced vital capacity (FVC) was $1.81 \pm 0.66 \mathrm{~L}(66 \% \pm 20 \%$ predicted). The three commonest referral diagnoses were ILD of uncertain classification $(n=38,39 \%)$, connective tissue disease-associated ILD (CTD-ILD) $(n=24,25 \%)$ and idiopathic pulmonary fibrosis (IPF) $(n=16,17 \%)$. Following evaluation by the ILD service, there was a change of diagnosis in $60(62 \%)$ patients and a change of management in 71 (73\%) patients. The majority of consensus MDM diagnoses were IPF $(n=35,36 \%)$, CTD-ILD $(n=30,30 \%)$ and others $(n=15,15 \%)$. There was a significant prognostic separation between the IPF and non-IPF diagnoses made following evaluation by the ILD service.

CONCLUSION The ILD service allowed for more precise subtyping of various ILDs. This is particularly useful for IPF patients, who can benefit from antifibrotic therapies.

Keywords: idiopathic interstitial pneumonia, idiopathic pulmonary fibrosis, interstitial lung disease, quality of health care

\section{INTRODUCTION}

Interstitial lung disease (ILD) is a heterogeneous group of diseases with varying disease behaviours and prognoses. Multidisciplinary meetings (MDMs) that involve chest clinicians, radiologists and pathologists are the current diagnostic standard for ILD. ${ }^{(1)}$ Flaherty et al first showed that multidisciplinary discussions involving the different subspecialties improve diagnostic performance in patients with ILDs. ${ }^{(2)}$ Two societies have recently published guidance on how an MDM should be conducted, ${ }^{(3,4)}$ with an ontological framework being developed to standardise the diagnostic classification of the different ILDs. ${ }^{(5}$

Diagnosing idiopathic pulmonary fibrosis (IPF), the most common form of idiopathic interstitial pneumonia (IIP), has become critically important, as the disease has a devastating prognosis with a median survival of 2.5-3.5 years. ${ }^{(6)}$ There are also management implications, as patients with IPF can now potentially be treated with new antifibrotic therapies such as pirfenidone and nintedanib, both of which were approved in Singapore in 2016 following two landmark trials published in $2014^{(7,8)}$ that showed a reduction in the decline of lung function in IPF patients. In addition, immunosuppression with a combination of prednisolone and azathioprine should be avoided, as it increases mortality and hospitalisation in this group of patients. ${ }^{(9)}$ In contrast, patients with certain types of connective tissue disease-associated interstitial lung disease (CTD-ILD), such as systemic sclerosis and idiopathic inflammatory myopathiesrelated ILDs, may benefit from systemic immunomodulatory therapies like cyclophosphamide and mycophenolate mofetil.

Studies on ILD MDM that showed positive diagnostic and management implications on patient care are predominantly from established ILD expert centres in the United States, ${ }^{(2)}$ Europe $^{(10,11)}$ and Australia. ${ }^{(12)}$ There is a paucity of such data from Southeast Asia. We performed a retrospective evaluation of our centre's ILD service to evaluate whether the positive impact of an ILD clinic and MDM on the diagnosis and management of ILDs demonstrated in overseas expert centres can be translated to our local setting. In addition, we validated the diagnostic accuracy of IPF in our ILD service against other non-IPF diagnoses.

\section{METHODS}

We are a university-affiliated tertiary hospital in Singapore with an ILD multidisciplinary team that comprises a chest physician, two thoracic radiologists, a rheumatologist and a pathologist, all of whom have received prior training from overseas expert ILD centres. In addition, we have a clinical pharmacist who provides disease and medication counselling. We performed a single-centre retrospective review of consecutive patients referred to the ILD clinic with subsequent discussions in ILD MDMs

\footnotetext{
${ }^{1}$ Department of Respiratory and Critical Care Medicine, Tan Tock Seng Hospital, ${ }^{2}$ Department of General Medicine, Khoo Teck Puat Hospital, ${ }^{3}$ Department of Diagnostic Radiology, ${ }^{4}$ Department of Pathology, ${ }^{5}$ Department of Pharmacy, ${ }^{6}$ Department of Rheumatology, Allergy and Immunology, Tan Tock Seng Hospital, Singapore 


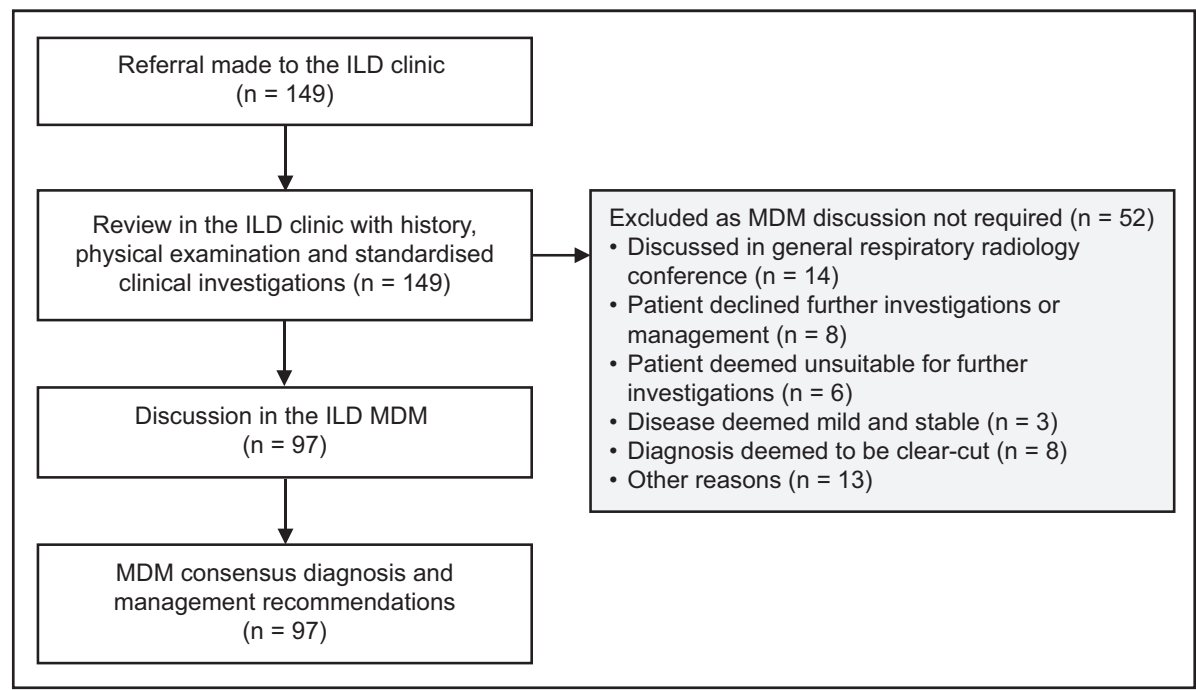

Fig. 1 Flowchart shows patients referred to the interstitial lung disease (ILD) clinic and subsequent multidisciplinary meeting (MDM) evaluation.

over an 18-month period from March 2016 to August 2017. All patients underwent evaluation with a complete history, physical examination and standardised investigations. The investigations included basic blood tests, lung function tests, high-resolution computed tomography of the chest, autoimmune serologies and serum brain natriuretic peptide levels. In selected patients, echocardiography, bronchoalveolar lavage, transbronchial lung biopsy or surgical lung biopsy (SLB) were performed.

Cases of suspected IPF, non-IPF fibrotic lung disease (such as fibrotic nonspecific interstitial pneumonia [NSIP], chronic hypersensitivity pneumonitis [HP] and sarcoidosis), ILDs that were not fully characterised and those with complex management issues were presented at our monthly ILD MDM. During the MDM, all the available clinical information was projected on a screen and the relevant thoracic radiology images were presented by the radiologists. The pathologist also presented selected biopsy images during the meeting. The referral diagnosis and management plan as stated in the referral letter were then compared against the consensus diagnosis that was made at the MDM. Diagnoses were classified according to the American Thoracic Society/European Respiratory Society IIP guidelines. ${ }^{(1)}$

We validated the diagnoses made in the ILD MDM by comparing the mortality of IPF against the other diagnoses. The survival period was calculated from the referral date to the ILD clinic to the date of death or 1 December 2017, which was the end of the study period. This study (no. 2017/00001) was reviewed by the National Healthcare Group Domain Specific Review Board (DSRB) and the need for DSRB review was deemed not necessary.

We used IBM SPSS Statistics version 20.0 for Windows (IBM Corp, Armonk, NY, USA) for all statistical analyses. Categorical data was displayed as absolute numbers and relative frequencies. Missing data was removed from the denominator when calculating relative frequencies. Continuous data was shown as mean \pm standard deviation for normally distributed data or as median (interquartile range [IQR]) for nonparametric data. Survival analysis was used to validate the MDM diagnosis. The Kaplan-Meier survival curve was utilised to show cumulative survival of IPF versus
non-IPF MDM diagnoses. Log-rank test for survival equality was used to compare survival curves. Cox regression analysis was used to evaluate the hazard ratio (95\% confidence interval [CI]) of MDM IPF diagnoses. Statistical significance was determined at $\mathrm{p}<0.05$.

\section{RESULTS}

A total of 149 patients were referred to the ILD clinic over an 18-month period from 1 March 2016 to 31 August 2017 (Fig. 1). Of these, 97 cases were discussed at the ILD MDM and included in this retrospective analysis. The remaining 52 cases did not require discussion at the MDM due to the reasons listed in Fig. 1. The baseline demographics and pulmonary physiology of the 97 included patients are illustrated in Table I. The mean age of the patients was $67 \pm 11$ years with $52 \%$ (50/97) male. With regard to the referral source, $58 \%$ (56/97) of the patients were referred from other respiratory physicians and 22\% (21/97) from rheumatologists. The majority of the patients were Chinese (79/97, $81 \%$ ) and never-smokers $(61 / 97,63 \%)$, and $14 \%$ (14/97) were on supplemental oxygen. The median duration of symptoms that patients experienced before they were seen at the ILD clinic was 12 (IQR 7-36) months. All patients had a moderately reduced restrictive ventilatory defect with a forced vital capacity (FVC) of $66 \% \pm 20 \%$ predicted. Diffusing capacity for carbon monoxide was moderately reduced at $52 \% \pm 16 \%$ predicted. The majority of the diagnoses $(65 / 97,67 \%)$ were based on clinico-radiological findings and MDM consensus, while $8(8 \%)$ patients needed histology from an SLB for a more accurate diagnosis. Of the remaining 24 patients who were considered for SLB, 38\% (9/24) were deemed too high risk for surgery, 29\% (7/24) declined surgery, and the rest were either too old or frail $(4 / 24,17 \%)$ or deemed unlikely to have a change of management (4/24, $17 \%$ ).

The referral diagnoses and MDM consensus diagnoses are shown in Fig. 2. Overall, evaluation by the ILD service (i.e. ILD clinic and MDM) resulted in a change in diagnosis in $62 \%(60 / 97)$ of the patients. The majority of the diagnoses that were stated in the referral letter (Fig. 2) were ILD with an uncertain classification (38/97, 39\%). The other diagnoses include CTD-ILD (24/97, 

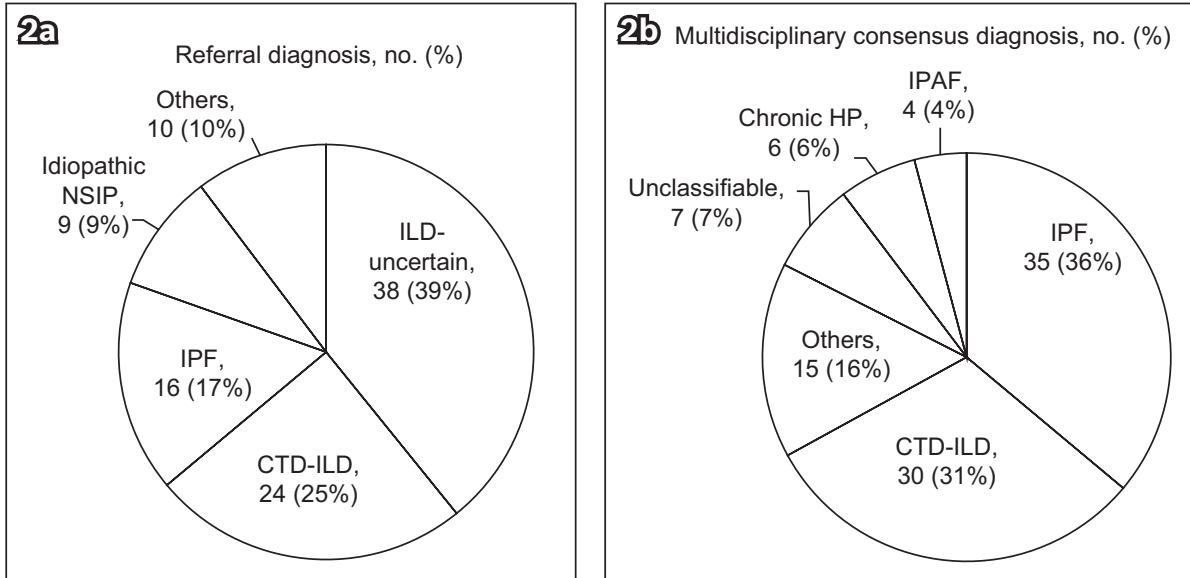

Fig. 2 Pie charts show (a) referral and (b) multidisciplinary consensus diagnoses. CTD-ILD: connective tissue disease-associated interstitial lung disease; HP: hypersensitivity pneumonitis; ILD: interstitial lung disease; IPAF: interstitial pneumonia with autoimmune features; IPF: idiopathic pulmonary fibrosis; NSIP: nonspecific interstitial pneumonia

Table I. Baseline demographics and pulmonary physiology $(n=97)$.

\begin{tabular}{|c|c|}
\hline Parameter & No. (\%) \\
\hline Age $^{*}(y r)$ & $67 \pm 11$ \\
\hline $\mathrm{BMI} *\left(\mathbf{k g} / \mathbf{m}^{2}\right)$ & $23.8 \pm 4.3$ \\
\hline Male gender & $50(52)$ \\
\hline Duration of symptoms ${ }^{\dagger}(\mathrm{mth})$ & $12(7-36)^{\ddagger}$ \\
\hline \multicolumn{2}{|l|}{ Ethnicity } \\
\hline Chinese & $79(81)$ \\
\hline Malay & $9(9)$ \\
\hline Indian & $8(8)$ \\
\hline Others & $1(1)$ \\
\hline \multicolumn{2}{|l|}{ Smoking history } \\
\hline Never & $61(63)$ \\
\hline Ex-smoker & $25(26)$ \\
\hline Current & $11(11)$ \\
\hline Use of supplemental oxygen & $14(14)$ \\
\hline \multicolumn{2}{|l|}{ Referral source } \\
\hline Respiratory medicine & $56(58)$ \\
\hline Rheumatology & $21(22)$ \\
\hline Others & $20(21)$ \\
\hline Surgical lung biopsy & $8(8)$ \\
\hline \multicolumn{2}{|l|}{ Pulmonary physiology* } \\
\hline $\mathrm{FVC}(\mathrm{L})(\mathrm{n}=94)$ & $1.81 \pm 0.66$ \\
\hline FVC (\% predicted) $(n=94)$ & $66 \pm 20$ \\
\hline $\operatorname{TLC}(\mathrm{L})(\mathrm{n}=80)$ & $3.43 \pm 0.99$ \\
\hline TLC (\% predicted) $(n=80)$ & $76 \pm 18$ \\
\hline DLCO $(\%$ predicted $)(n=74)$ & $52 \pm 16$ \\
\hline
\end{tabular}

*Data presented as mean \pm standard deviation. †Data presented as median (interquartile range). $\neq$ Duration of symptoms was calculated from 89 patients, as 8 patients were asymptomatic. BMI: body mass index; DLCO: diffusing capacity for carbon monoxide; FVC: forced vital capacity; TLC: total lung capacity

$25 \%), \operatorname{IPF}(16 / 97,17 \%)$ and idiopathic NSIP (9/97, 9\%). The most common diagnosis after MDM evaluation was IPF (35/97, $36 \%)$, followed by CTD-ILD (30/97, 31\%) i.e. an additional 19 cases of IPF and six cases of CTD-ILD. Following evaluation by the ILD clinic and MDM, we reclassified all 38 ILDs with an uncertain classification, with the majority (20/38, 53\%) having an eventual diagnosis of IPF. Of note, of the 16 patients with a referral diagnosis of IPF, a small minority of the patients (5/16, $31 \%$ ) turned out to have an alternative diagnosis other than IPF. All patients with a referral diagnosis of NSIP were reclassified as IPF $(n=2)$, CTD-ILD $(n=2)$, chronic HP $(n=3)$ and unclassifiable ILD $(n=2)$. Additional diagnoses of chronic HP and interstitial pneumonia with autoimmune features were also made after evaluation by the ILD clinic and MDM. The change in diagnosis was predominantly seen in referrals from respiratory medicine and other disciplines (70\% and 85\%, respectively; Table II).

The MDM diagnoses of IPF versus the non-IPF diagnoses were validated in the univariate Cox regression analysis, and the Kaplan-Meier survival curve is shown in Fig. 3. Median followup time was 11 (IQR 7-16.5) months. There was significant prognostic separation with a hazard ratio of $5.07(95 \% \mathrm{Cl}$ $1.69-15.21, p=0.004)$.

Overall, $71(73 \%)$ patients had a change in management after evaluation by the ILD clinic and MDM. The majority (49/97, 51\%) were directly due to a change in diagnosis (Table III). Of the 49 patients from this group, the main recommendations included initiating antifibrotic therapy $(\mathrm{n}=21)$ and immunomodulatory therapy $(n=19)$. The change in management occurred across all referral sources, which ranged from $57 \%$ of rheumatology referrals and $75 \%$ of referrals from other specialties (Table II).

\section{DISCUSSION}

In this retrospective single-tertiary-centre analysis, a dedicated ILD service comprising an ILD clinic and MDM resulted in changes in diagnosis and subsequent management in the majority of the cohort. In addition, the accuracy of IPF diagnosis was verified by the significant prognostic separation of an IPF diagnosis versus the non-IPF ones. This compares favourably to the studies from Australia and the United Kingdom (UK) in which $53 \%{ }^{(12)}$ and $76 \%{ }^{(11)}$ of the patients, respectively, had a change in diagnosis after evaluation by an ILD multidisciplinary service. In the UK study, $40 \%$ of the patients also had their management altered after ILD multidisciplinary evaluation. ${ }^{(11)}$ 


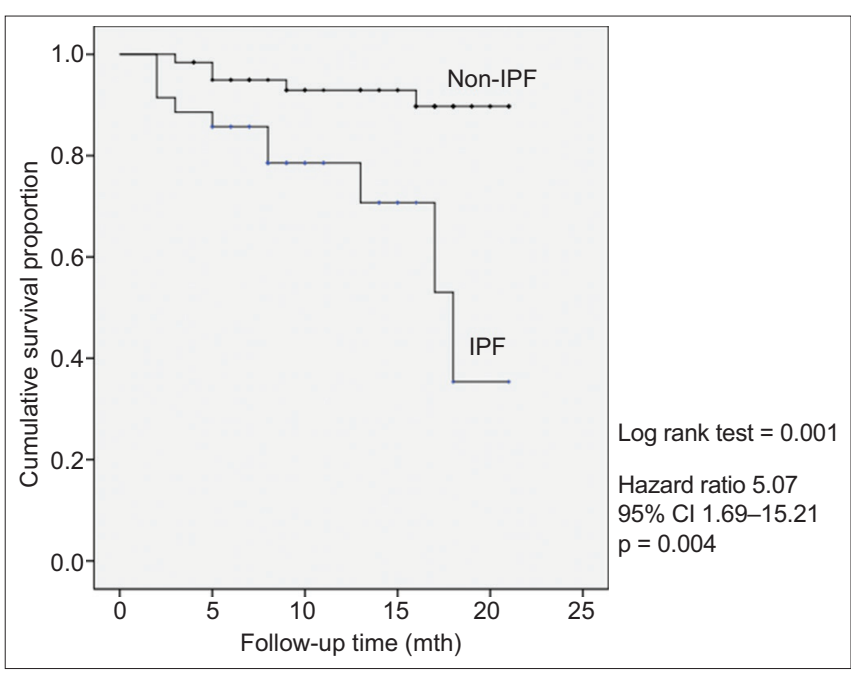

Fig. 3 Kaplan-Meier curve shows survival difference between patients with idiopathic pulmonary fibrosis (IPF) and those without IPF. Median follow-up time was 11 (interquartile range $7-16.5$ ) months. $\mathrm{Cl}$ : confidence interval

Table II. Change in diagnosis and management according to referral source.

\begin{tabular}{|lll|}
\hline Referral source & \multicolumn{2}{c|}{ No. (\%) } \\
\cline { 2 - 3 } & $\begin{array}{l}\text { Change in } \\
\text { diagnosis }\end{array}$ & $\begin{array}{l}\text { Change in } \\
\text { management }\end{array}$ \\
\hline Respiratory medicine $(n=56)$ & $39(70)$ & $46(82)$ \\
\hline Rheumatology $(n=21)$ & $4(19)$ & $12(57)$ \\
\hline Others* $(n=20)$ & $17(85)$ & $13(65)$ \\
\hline
\end{tabular}

*Others included general medicine $(n=6)$, cardiology $(n=5)$, infectious disease $(n=2)$, primary care $(n=2)$, neurology $(n=2)$, gastroenterology $(n=1)$, geriatric medicine $(n=1)$ and otolaryngology $(n=1)$.

Table III. Changes in management after ILD clinic evaluation and MDM discussion.

\begin{tabular}{|ll|}
\hline Post-ILD clinic evaluation and MDM discussion & No. (\%) \\
\hline Change in diagnosis with change in management & $49(51)$ \\
\hline Initiate antifibrotic therapy & $21(43)$ \\
\hline Initiate immunosuppression & $19(39)$ \\
\hline Augment immunosuppression & $3(6)$ \\
\hline Others & $6(12)$ \\
\hline Change in diagnosis but no change in management & $11(11)$ \\
\hline No change in diagnosis but change in management & $22(23)$ \\
\hline Initiate antifibrotic therapy & $13(59)$ \\
\hline Initiate immunosuppression & $1(5)$ \\
\hline Augment immunosuppression & $4(18)$ \\
\hline Others & $4(18)$ \\
\hline No change in diagnosis and no change in management & $15(15)$ \\
\hline
\end{tabular}

ILD: interstitial lung disease; MDM: multidisciplinary meeting

In the present study, the patients who presented to our ILD service had more severe lung disease compared to other similar cohorts. Using FVC as an indicator of disease severity, the mean FVC in our study was $66 \%$ predicted, which is lower than the Australian (FVC $77 \%$ predicted) ${ }^{(12)}$ and Danish (FVC $71 \%$ predicted) cohorts. ${ }^{(13)}$ Also, $14 \%$ of our patients were on supplemental oxygen, indicating underlying chronic hypoxaemic respiratory failure or significant exercise desaturation. We speculate that patients presented to our ILD service later in their disease course due to delayed recognition and referral to the appropriate specialists. Indeed, the median duration of symptoms experienced by patients before attendance at the ILD service was one year; this is similar to a European survey of IPF patients where $58 \%$ of the respondents reported a delay of more than one year between initial presentation and definite diagnosis of IPF. ${ }^{(14)}$ Hence, further improvement in educating physicians to recognise ILDs is needed so that appropriate referrals can be made promptly.

The main referral diagnosis we received was ILD of an uncertain classification. This mirrors the retrospective analysis of an ILD multidisciplinary service done in the UK ${ }^{(11)}$ where $23 \%$ of the ILDs referred were not classified. In our study, the majority of these patients had an eventual diagnosis of IPF. It must be emphasised that the diagnosis of IPF carries major prognostic and management implications. Similarly, patients with underlying CTD-ILD may experience the pulmonary and extra-pulmonary benefits of immunomodulatory treatment, which can control disease activity and prevent progressive organ damage. With ready access to a service that comprises an ILD clinic and MDM, it will no longer be acceptable to label patients with an umbrella term of ILD without further classification, thereby subjecting them to diagnostic and prognostic uncertainties. This may ultimately lead to inappropriate or missed treatment opportunities. ${ }^{(15)}$

Our SLB rates were much lower than that of the Danish cohort $(40 \%)^{(13)}$ but higher than that of the UK cohort $\left.(5 \%)\right)^{(11)}$ This may reflect a change in practice over the years, with more widespread adoption of ILD MDMs so that clinical data, radiological patterns, bronchoalveolar lavage, disease course and treatment outcomes ${ }^{(16)}$ are integrated and discussed among the various specialties. This is reflected in the falling rates of SLB for the diagnosis of IPF; in earlier studies, SLB rates as high as $65 \%$ were reported in the 2004 GIPF-001 study ${ }^{(17)}$ versus the more recent 2014 INPULSIS study, where only $22 \%$ of the patients underwent SLB.$^{(8)}$ Close to half of our patients were unable to undergo SLB either because they were too old or frail, or the procedure was deemed too high risk. Indeed, SLB carries a 30day mortality of $2.4 \%$ based on a hospital statistics database from 1997 to 2008 in England, UK. ${ }^{(18)}$ This is comparable to the $2.3 \%$ 30-day mortality for lobectomy for non-small cell lung cancer, ${ }^{(19)}$ a potentially curative procedure rather than a diagnostic one. Looking forward, transbronchial lung cryobiopsy, which offers an overall diagnostic yield of $81 \%$ with lower complication and mortality rates than $\mathrm{SLB},{ }^{(20)}$ may be considered in patients who are unfit for SLB.

Regarding the diagnosis of IPF, only 11 of the 16 patients referred for IPF had an eventual consensus diagnosis of IPF. The ILD clinic and MDM also diagnosed an additional 24 patients with IPF. Even though a recent study, whose participants comprised expert ILD clinicians, suggested that clinicians can diagnose IPF with similar levels of accuracy as their respective MDMs, ${ }^{(10)}$ our results clearly showed that this cannot be replicated in a broader group of chest physicians, each with different levels of experience. Indeed, a subsequent international study evaluating the diagnostic accuracy of a clinical diagnosis of IPF without a multidisciplinary 
evaluation found that only clinicians with more than 20 years of experience and those with regular MDM attendance were able to diagnose IPF with similar accuracy as IPF experts. ${ }^{(21)}$

With regard to the distribution of MDM diagnosis, IPF was the most common diagnosis. This is consistent with the findings of the Australian ${ }^{(12)}$ and Danish ${ }^{(13)}$ cohorts. The second most common diagnosis was CTD-ILD, accounting for $30 \%$ of the diagnoses. The proportion of this diagnosis is higher compared to the Australian study, ${ }^{(12)}$ the Indian ILD registry ${ }^{(22)}$ and the Denmark ILD cohort, ${ }^{(13)}$ because our centre has a large affiliated rheumatology department. ${ }^{(23)}$ On the contrary, chronic HP and unclassifiable ILD were less frequent in our cohort as compared to other cohorts. The diagnosis of chronic HP is often challenging and $50 \%$ or more of patients may not have a clear exposure history. ${ }^{(24)}$ Unlike IPF, there is a lack of consensus guidelines on how this disease is diagnosed and the diagnostic agreement among expert multidisciplinary teams is poor (weighted kappa value of 0.29$).{ }^{(10)}$ With the absence of local data, it remains uncertain if the low frequency represents a true low incidence of this entity in our country or underdiagnosis. The frequency of unclassifiable ILD in our cohort was only $7 \%$, which is lower than the reported frequency of $10 \%-14 \% ;{ }^{(13,25)}$ this may be because our referred cases had less complexity than other cohorts that evaluated patients in designated specialised ILD centres. ${ }^{(12,13)}$

Idiopathic NSIP is a very rare condition, and none of our patients had this diagnosis. Indeed, following rigorous multidisciplinary evaluation by expert clinicians, radiologists and pathologists of an American Thoracic Society Workgroup, only 67 definite or probable idiopathic NSIP patients were identified among 305 patients who were previously reported to have idiopathic NSIP; the majority of the NSIP cases excluded by this workgroup were $\mathrm{HP}$, usual interstitial pneumonia and organising pneumonia. ${ }^{26}$ Additionally, NSIP may be a manifestation of an underlying CTD. ${ }^{(27)}$ In our study, patients referred for idiopathic NSIP were reassigned a diagnosis of CTD-ILD, chronic HP, IPF or unclassifiable ILD. This is similar to the existing published literature.

The use of the survival difference between IPF and non-IPF to validate the diagnostic accuracy of IPF at our MDM warrants further discussion. IPF carries a very poor prognosis, which differentiates this disease from other ILDs. In fact, its five-year survival is worse than the majority of malignancies except lung and pancreatic cancers. ${ }^{(28)}$ Median survival is 2.5-3.5 years, ${ }^{(6)}$ and only $21 \%$ of the patients demonstrate a slowly progressive course. ${ }^{(29)}$ The survival is markedly worse compared to other ILDs such as chronic HP (median survival seven years) ${ }^{(30)}$ and idiopathic NSIP (five-year survival 82.3\%). ${ }^{(26)}$ Similarly, in systemic sclerosis-related ILD, one of the most commonly seen CTD-ILDs, the median survival is $5-8$ years. ${ }^{(31)}$ This method has been used to evaluate the diagnostic accuracy of IPF in an international ILD MDM study. ${ }^{(10)}$ The significant prognostic separation between the diagnosis of IPF and other ILDs validates our MDM evaluation in terms of the diagnostic accuracy of IPF, which is the commonest IIP.

Almost three-quarters of our patients had a change in management after the ILD clinic evaluation; this was directly related to more accurate diagnoses in the majority of patients. Management recommendations included initiating antifibrotic or immunomodulatory therapy. There were also a significant number of patients with a change in management without any change in diagnosis, with the predominant treatment recommendation being initiation of antifibrotic therapy. This may be due to general chest physicians' lack of familiarity with the use of recently approved antifibrotic therapies, as well as the prohibitive costs of these therapies locally. A similar finding is shown in a survey of Canadian academic and community chest physicians or trainees, where only $34 \%$ of the responders used the antifibrotic pirfenidone in patients with IPF. ${ }^{(32)}$

The majority of changes in diagnosis occurred in referrals from respiratory medicine and other disciplines. While it is not surprising to see a high frequency of diagnosis changes among patients referred from other non-respiratory disciplines, it is surprising that almost three-quarters of the referrals from respiratory medicine had a change in diagnosis following evaluation by the ILD service. This may be because the clinicians were unfamiliar with what to look out for in the diagnosis of ILD. Just looking at IPF alone, the commonest of the IIPs, one survey of French pulmonologists found that one-third of the respondents were not aware of the 2011 IPF international guidelines. ${ }^{(33)}$ In another case cohort study, individual academic physicians needed more than 20 years of experience in order to diagnose IPF at a level achieved by expert ILD clinicians. ${ }^{(21)}$ In our study, among the referrals from rheumatologists, a diagnosis of CTD-ILD was much less of an issue; however, after ILD service evaluation, there was a change of management in $57 \%$ of the referrals. Although this is lower than the results reported by Castelino et al, ${ }^{(34)}$ their findings must be interpreted in the context of a much higher rate of lung biopsy $(40 \%)$, which resulted in a change in diagnosis or treatment in $50 \%$ of them. It is uncertain if we can achieve similar rates of treatment change if our cohort were to have such a high lung biopsy rate.

There are a few limitations in our study. Firstly, this is a single-centre retrospective study with a small sample size, and the diagnostic accuracy of the MDM can only be validated by the prognostic difference of IPF compared to the non-IPF entities. The logical next step is for an expert centre to validate the consensus MDM diagnosis, a method that was employed by the ILD registry in India. ${ }^{(22)}$ Secondly, the magnitude of the change in diagnosis and management is likely to be overestimated, as the majority of the referral diagnoses were ILD of an uncertain classification. Nonetheless, the ILD service has achieved its aim in providing a more precise diagnosis and management for this group of patients, who would have otherwise been labelled with an uncertain ILD diagnosis. Thirdly, the length of follow-up is relatively short and, consequently, the clinical impact of the change in diagnosis and management cannot be fully characterised. Fourthly, there may be referral bias, as patients who were deemed unfit for antifibrotic therapy or immunomodulation may not have been referred to the ILD service; also, a minority of patients from the ILD clinic were discussed at the general respiratory radiology meeting and not the ILD MDM. Finally, our SLB rates were lower than those 
reported in the published literature, ${ }^{(13)}$ and so it is uncertain if a greater magnitude of change in diagnosis or management can be achieved with higher biopsy rates.

In conclusion, this is the first study in Southeast Asia to evaluate the clinical impact of a dedicated ILD clinic and MDM. A significant number of patients had a change in diagnosis with subsequent change in management. Importantly, the group with ILD of uncertain classification were subtyped more accurately, with the majority eventually classified as IPF. Patients usually present late in their disease course, so it is important that physicians identify these patients early, so that appropriate referral can be made and specific therapies instituted promptly. Although long-term outcomes remain to be seen, a multidisciplinary approach to diagnosis and management of ILDs should be the standard of care for this group of patients.

\section{ACKNOWLEDGEMENTS}

The authors thank Dr Tan Geak Poh and his staff at the respiratory function laboratory, Tan Tock Seng Hospital, Singapore, for their invaluable input to the manuscript and for providing the pulmonary function results. All authors report no conflict of interests.

\section{REFERENCES}

1. Travis WD, Costabel U, Hansell DM, et al; ATS/ERS Committee on Idiopathic Interstitial Pneumonias. An official American Thoracic Society/European Respiratory Society statement: Update of the international multidisciplinary classification of the idiopathic interstitial pneumonias. Am J Respir Crit Care Med 2013; 188:733-48.

2. Flaherty KR, King TE Jr, Raghu G, et al. Idiopathic interstitial pneumonia: what is the effect of a multidisciplinary approach to diagnosis? Am J Respir Crit Care Med 2004; 170:904-10.

3. Lynch DA, Sverzellati N, Travis WD, et al. Diagnostic criteria for idiopathic pulmonary fibrosis: a Fleischner Society White Paper. Lancet Respir Med 2018; 6:138-53.

4. Prasad JD, Mahar A, Bleasel J, et al. The interstitial lung disease multidisciplinary meeting: A position statement from the Thoracic Society of Australia and New Zealand and the Lung Foundation Australia. Respirology 2017; 22:1459-72.

5. Ryerson CJ, Corte TJ, Lee JS, et al. A standardized diagnostic ontology for fibrotic interstitial lung disease. An international working group perspective. Am J Respir Crit Care Med 2017; 196:1249-54.

6. Ley B, Collard HR, King TE Jr. Clinical course and prediction of survival in idiopathic pulmonary fibrosis. Am J Respir Crit Care Med 2011; 183:431-40.

7. King TE Jr, Bradford WZ, Castro-Bernardini S, et al; ASCEND Study Group. A phase 3 trial of pirfenidone in patients with idiopathic pulmonary fibrosis. $\mathrm{N}$ Engl J Med 2014; 370:2083-92.

8. Richeldi L, du Bois RM, Raghu G, et al; INPULSIS Trial Investigators. Efficacy and safety of nintedanib in idiopathic pulmonary fibrosis. N Engl J Med 2014; 370:2071-82.

9. Idiopathic Pulmonary Fibrosis Clinical Research Network, Raghu G, Anstrom KJ, et al. Prednisone, azathioprine, and $\mathrm{N}$-acetylcysteine for pulmonary fibrosis. $\mathrm{N}$ Engl J Med 2012; 366:1968-77.

10. Walsh SLF, Wells AU, Desai SR, et al. Multicentre evaluation of multidisciplinary team meeting agreement on diagnosis in diffuse parenchymal lung disease: a case-cohort study. Lancet Respir Med 2016; 4:557-65.

11. Chaudhuri N, Spencer L, Greaves M, et al. A review of the multidisciplinary diagnosis of interstitial lung diseases: a retrospective analysis in a single UK specialist centre. J Clin Med 2016; 5(8). pii: E66.

12. Jo HE, Glaspole IN, Levin KC, et al. Clinical impact of the interstitial lung disease multidisciplinary service. Respirology 2016; 21:1438-44.

13. Hyldgaard C, Hilberg O, Muller A, Bendstrup E. A cohort study of interstitial lung diseases in central Denmark. Respir Med 2014; 108:793-9.

14. Schoenheit G, Becattelli I, Cohen AH. Living with idiopathic pulmonary fibrosis: an in-depth qualitative survey of European patients. Chron Respir Dis 2011; 8:225-31.

15. Troy L, Glaspole I, Goh N, et al. Prevalence and prognosis of unclassifiable interstitial lung disease. Eur Respir J 2014; 43:1529-30.

16. Cottin V. Lung biopsy in interstitial lung disease: balancing the risk of surgery and diagnostic uncertainty. Eur Respir J 2016; 48:1274-7.

17. Raghu G, Brown KK, Bradford WZ, et al; Idiopathic Pulmonary Fibrosis Study Group. A placebo-controlled trial of interferon gamma-1b in patients with idiopathic pulmonary fibrosis. N Engl J Med 2004; 350:125-33.

18. Hutchinson JP, McKeever TM, Fogarty AW, Navaratnam V, Hubbard RB. Surgical lung biopsy for the diagnosis of interstitial lung disease in England: 1997-2008. Eur Respir J 2016; 48:1453-61.

19. Powell HA, Tata LJ, Baldwin DR, et al. Early mortality after surgical resection for lung cancer: an analysis of the English National Lung cancer audit. Thorax 2013; 68:826-34.

20. Ravaglia C, Bonifazi M, Wells AU, et al. Safety and diagnostic yield of transbronchial lung cryobiopsy in diffuse parenchymal lung diseases: a comparative study versus video-assisted thoracoscopic lung biopsy and a systematic review of the literature. Respiration 2016; 91:215-27.

21. Walsh SLF, Maher TM, Kolb M, et al; IPF Project Consortium. Diagnostic accuracy of a clinical diagnosis of idiopathic pulmonary fibrosis: an international case-cohort study. Eur Respir J 2017; 50(2). pii: 1700936.

22. Singh S, Collins BF, Sharma BB, et al. Interstitial lung disease in India: results of a prospective registry. Am J Respir Crit Care Med 2017; 195:801-13.

23. Koh ET, Tan JW, Thong BY, et al; TTSH RA Study Group. Major trends in the manifestations and treatment of rheumatoid arthritis in a multiethnic cohort in Singapore. Rheumatol Int 2013; 33:1693-703.

24. Salisbury ML, Myers JL, Belloli EA, et al. Diagnosis and treatment of fibrotic hypersensitivity pneumonia: where we stand and where we need to go. Am J Respir Crit Care Med 2017; 196:690-9.

25. Ryerson CJ, Urbania TH, Richeldi L, et al. Prevalence and prognosis of unclassifiable interstitial lung disease. Eur Respir J 2013; 42:750-7.

26. Travis WD, Hunninghake G, King TE Jr, et al. Idiopathic nonspecific interstitial pneumonia: report of an American Thoracic Society project. Am J Respir Crit Care Med 2008; 177:1338-47.

27. Wells AU, Cottin V. Nonspecific interstitial pneumonia: time to be more specific? Curr Opin Pulm Med 2016; 22:450-5.

28. Vancheri C, Failla M, Crimi N, Raghu G. Idiopathic pulmonary fibrosis: a disease with similarities and links to cancer biology. Eur Respir J 2010; 35:496-504.

29. Fernández Pérez ER, Daniels CE, Schroeder DR, et al. Incidence, prevalence, and clinical course of idiopathic pulmonary fibrosis: a population-based study. Chest 2010; 137:129-37.

30. Vasakova M, Morell F, Walsh S, Leslie K, Raghu G. Hypersensitivity pneumonitis: perspectives in diagnosis and management. Am J Respir Crit Care Med 2017; 196:680-9.

31. Altman RD, Medsger TA Jr, Bloch DA, Michel BA. Predictors of survival in systemic sclerosis (scleroderma). Arthritis Rheum 1991; 34:403-13.

32. Weatherald J, McFadden R, Fell CD. Trends in diagnosis and management of idiopathic pulmonary fibrosis in Canada. Can J Respir Crit Care Sleep Med 2017; 1:71-6.

33. Cottin V, Cadranel J, Crestani B, et al. Management of idiopathic pulmonary fibrosis in France: a survey of 1244 pulmonologists. Respir Med 2014; 108:195-202.

34. Castelino FV, Goldberg H, Dellaripa PF. The impact of rheumatological evaluation in the management of patients with interstitial lung disease. Rheumatology (Oxford) 2011; 50:489-93. 